Wandel in der Strahlentherapie zu weniger invasiver Therapie möglich

\section{Intraoperative Radiotherapie mit INTRABEAM $^{\circledR}$ als Methode zur Brustkrebsbehandlung anerkannt}

Führende Wissenschaftler und Ärzte bestätigen die intraoperative Radiotherapie (IORT) mit dem INTRABEAM ${ }^{\circledR}$ von Carl Zeiss Meditec als neue Alternative zur heutigen Strahlentherapie bei der Brustkrebsbehandlung. $\mathrm{Zu}$ diesem Ergebnis kam ein Symposium, das sich mit dem Stand der Technologieentwicklung der IORT und ihrer Implementierung in die klinische Praxis beschäftigte. Die gezielte intraoperative Bestrahlung des betroffenen $\mathrm{Ge}$ webes im Tumorbett mit dem INTRABEAM schont das umliegende gesunde Gewebe, ist daher weniger aggressiv und bietet so einen klaren Behandlungsvorteil für die Patientinnen; zusätzlich reduziert sich die Behandlungsdauer.

Mit dem INTRABEAM führt Carl Zeiss Meditec nach langer Entwicklungszeit ein System in den Markt ein, dessen Behandlungsvorteil für die Patientinnen bei der Brustkrebstherapie durch zahlreiche klinische Daten nachgewiesen ist - und von führenden Wissenschaftlern sowie praktizierenden Ärzten bestätigt wurde.

Die Applikation ist als Option in die «Practical Guidelines» der Deutschen Gesellschaft für Radioonkologie (DEGRO) aufgenommen worden. Dieser Aspekt sowie die unkomplizierte Bedienung führten dazu, dass die IORT mit dem INTRABEAM nicht mehr nur an Universitäten und Forschungseinrichtungen angewandt wird, sondern auch für große und kleinere Brustzentren von höchstem Interesse ist und dort zunehmend zum Einsatz kommt.
Carl Zeiss Meditec verfolgt mit der viel versprechenden Zukunftstechnologie des INTRABEAM konsequent das Ziel einer risikoadaptierten, weniger aggressiven Therapieoption zur Behandlung des Mammakarzinoms. Da für den Betrieb des Systems keine baulichen Veränderungen für Strahlenschutzmaßnamen notwendig sind und es sehr einfach zu bedienen ist, lässt es sich ohne großen Aufwand in den klinischen Ablauf implementieren. Bereits heute verfügen etwa $10 \%$ aller zertifizierten Brustzentren in Deutschland über die Möglichkeit der IORT mit INTRABEAM.

Weitere Informationen bei

Carl Zeiss Meditec AG

Eva Sesselmann

Göschwitzer Straße 51-52, 07745 Jena

Tel. +49 3641 220-331, Fax -112

press@meditec.zeiss.com

\section{Meningeosis neoplastica beim Mammakarzinom - ein Notfall!}

Die Meningeosis neoplastica (MN) beim Mammakarzinom führt unbehandelt innerhalb weniger Wochen zum Tod. Erhöhte Aufmerksamkeit ist angezeigt bei Kopfschmerzen, Rückenschmerzen, fokalneurologischen Veränderungen und Wesensveränderungen. Es muss eine sofortige Kontrastmittel-Magnetresonanztomografie (MRT) von Kopf und Wirbelsäule erfolgen. Eine gesicherte Diagnose erhält man mittels Liquorzytologie.

Entscheidungskriterien für die Wahl der Therapie sind der Allgemeinzustand und die Lebenserwartung der Patientin sowie das leptomeningeale Befallsmuster. Während bei der MN vom soliden Typ Strahlentherapie und systemische Chemotherapie im Vordergrund stehen, spielt bei der MN vom nonadhärenten Typ die intrathekale Chemotherapie in Kombination mit Bestrahlung und/oder Chemotherapie eine wichtige Rolle. Unverzichtbar ist auch eine adäquate supportive Therapie zur Symptomkontrolle. Zur intrathekalen Therapie hat sich in den letzten Jahren liposomales Cytarabin etabliert, das lumbal appliziert werden kann und das aufgrund seiner langen Halbwertszeit nur 1-mal alle 2 Wochen gegeben werden muss. In einer prospektivrandomisierten Studie erwies es sich als ebenso effektiv wie Methotrexat bei einfacherer Applikation und mit verlängerter Zeit bis zur neurologischen Progression und längerem Meningeosis-spezifischem Überleben. Ebenso verbessert liposomales Cytarabin das Lebensqualitäts-adjustierte Überleben.
Derzeit werden in interdisziplinären Studienkonzepten bei Patientinnen mit MN die Kombination von systemischer Therapie mit der intrathekalen Gabe von liposomalem Cytarabin bei HER2-positiven und bei HER2-negativen Patientinnen geprüft.

Quelle

Symposium am 11. Juni 2009, anlässlich der Jahrestagung der Deutschen Gesellschaft für Senologie, unterstützt von Mundipharma

Weitere Informationen bei

POMME-med GmbH

Steffi Weiss

Von-Erckert-Str. 48, 81827 München

Tel. +49 89 454566-24, Fax -27

weiss@pomme-med.de

\title{
PharmaTicker+++ PharmaTicker+++ PharmaTicker+++ PharmaTicker+++
}

Lilly Deutschland GmbH. Die von Eli Lilly and Company ins Leben gerufene Lilly Phenotypic Drug Discovery Initiative $\left(\mathrm{PD}^{2}\right)$ fördert den globalen Austausch zwischen Wissenschaft und Unternehmen. Weitere Informationen zu dieser neuen Forschungsinitiative sind unter www.pd2.lilly.com zu finden.

Lilly Deutschland $\mathrm{GmbH}$

Katrin Blank

Tel. +49 6172 273-2738, Fax -2539

pressestelle@lilly.com
Wyeth Pharma GmbH. Die Opioid-induzierte Obstipation (OIC) ist eine häufige Nebenwirkung der Opioid-Therapie, die für Betroffene einen wesentlichen Belastungsfaktor darstellt. Mit der neuen Broschüre «Verstopfung als Folge einer Opioid-Therapie» können Ärzte gezielt Patienten und deren Angehörige informieren. Ärzte können die Broschüre kostenlos auf der Website http://www.relistor.de herunterladen oder per Post bestellen.

Wyeth Pharma GmbH Mirja Kaupmann

Tel. +49 251 204-1226, Fax -2033

kaupmaM@wyeth.com
Cefak KG. Ausführliche Informationen zur Schutzwirkung von Selen auch in Bezug auf Chemo- und Strahlentherapie sowie bei Lymphödemen und in der Nachsorge finden Sie in der neuen Patientenbroschüre «Selen und gynäkologische Tumore». Die kostenlose Broschüre kann angefordert werden bei:

Cefak KG

Cefasel-Service

Tel. +49 831 57401-0, Fax -50

cefak@cefak.com

www.selen-info.de 